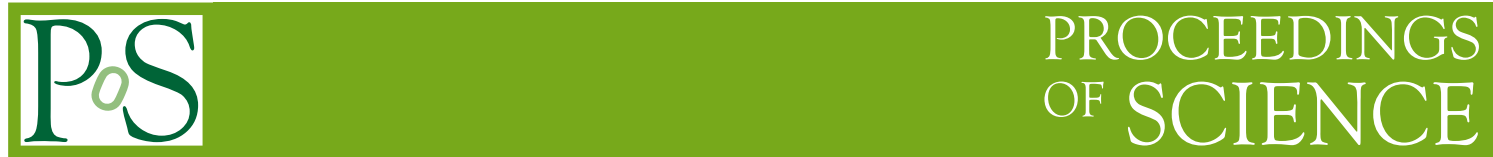

\title{
Particle ratio fluctuations in NA49
}

\section{Christof Roland for the NA49 Collaboration*}

Massachusetts Institute of Technology, Cambridge, USA

E-mail: Christof.Roland@cern.ch

The results of the NA49 energy scan program show a sharp maximum of the ratio of $K^{+}$to $\pi^{+}$ yields in central $\mathrm{Pb}+\mathrm{Pb}$ collisions at beam energies of 20-30 AGeV. This observation has been interpreted as an indication of a phase transition at low SPS energies. We present results on energy dependence of event-by-event fluctuations of the kaon to pion and proton to pion ratios at beam energies close to this maximum and complement this study with preliminary data from the STAR collaboration in the RHIC energy range. A significant increase of the fluctuation signal of the kaon to pion ratio at 20 and $30 \mathrm{AGeV}$ is observed while it stays constant from the highest SPS energies out to the RHIC energy range.

The 2nd edition of the International? / Workshop - Correlations and Fluctuations in Relativistic Nuclear Collisions -

July 7-9 2006

Galileo Galilei Institute, Florence, Italy

\footnotetext{
*Speaker.
} 


\section{Introduction}

Heavy-ion collisions at relativistic energies are investigated to study the creation and properties of a deconfined state of quarks and gluons, the quark gluon plasma (QGP). The key difficulty of the search for the QGP is the identification of experimental signatures that are directly sensitive to its creation. One proposed strategy is a study of the energy dependence of pion and strangeness yields $[1,2,3]$. The transition to the QGP is suggested to lead to anomalies in this dependence.

The energy scan program of the NA49 experiment at the CERN SPS recorded and analyzed $\mathrm{Pb}+\mathrm{Pb}$ collisions at beam energies of $20,30,40,80$ and $158 \mathrm{AGeV}$. The energy dependence of the ratio of $K^{+}$to $\pi^{+}$yields $[4,5]$ shown in figure 1 exhibits a non-monotonic behavior, with a sharp maximum close to $30 \mathrm{AGeV}$ and an indication of a nearly constant value at higher energies. This characteristic energy dependence is not observed in elementary interactions and seems to be an unique feature of heavy ion collisions. The measured dependence can be related to an increase of the entropy production and a decrease of the strangeness to entropy ratio in central $\mathrm{Pb}+\mathrm{Pb}$ collisions in the low SPS energy range. They can be understood within the Statistical Model of the Early Stage of nucleus-nucleus collisions, which assumes that a transient state of deconfined matter is created in $\mathrm{Pb}+\mathrm{Pb}$ collisions for energies larger than about $40 \mathrm{AGeV}[2,3]$. While this experimental result is consistent with a transition to deconfined matter, it does not reveal properties of the phase transition itself. It was realized however, that event-by-event fluctuations of particle ratios related to the hadro-chemical composition of the particle source could provide a direct probe of the existence and nature of the phase transition [6].

The proposed mechanisms that can lead to such event-by-event fluctuations are manifold and range from overheating-supercooling fluctuations due to a first order phase transition with large latent heat, over fluctuations due to a phase of coexisting confined and deconfined matter (mixed phase) in varying relative abundances, to true critical fluctuations in the vicinity of the critical point in the QCD phase diagram $[7,8,9,10]$.

Thus a scan of event-by-event observables over a wide range of collision scenarios, probing the properties of nuclear matter in various regions of the $\mu_{B}$ vs $\mathrm{T}$ phase diagram, is performed to provide information directly related to the nature of the phase transition. Experimentally the energy density achieved in the reaction can be controlled by varying the incident energy of the colliding system. In this article, the energy dependence of the event-by-event particle ratios $\left[K^{\dagger}+\right.$ $\left.K^{-}\right] /\left[\pi^{+}+\pi^{-}\right]$and $[p+\bar{p}] /\left[\pi^{+}+\pi^{-}\right]$are presented.

\section{Experimental Setup and Data Selection}

The NA49 experiment was conceived to measure event-by-event fluctuations in heavy ion collisions. The NA49 large acceptance hadron spectrometer allows the detection of more than 1000 individual charged particles for a single central $\mathrm{Pb}+\mathrm{Pb}$ collision and is well suited for a measurement of particle ratios in single $\mathrm{Pb}+\mathrm{Pb}$ collisions at all available beam energies. A detailed description of the NA49 experiment can be found in [11].

The charged particle reconstruction in this analysis is based on the trajectory measured in the large volume Time Projection Chambers (TPC). In addition to the momentum determination through the curvature in the magnetic field the TPC's allow to identify particles by measuring the 


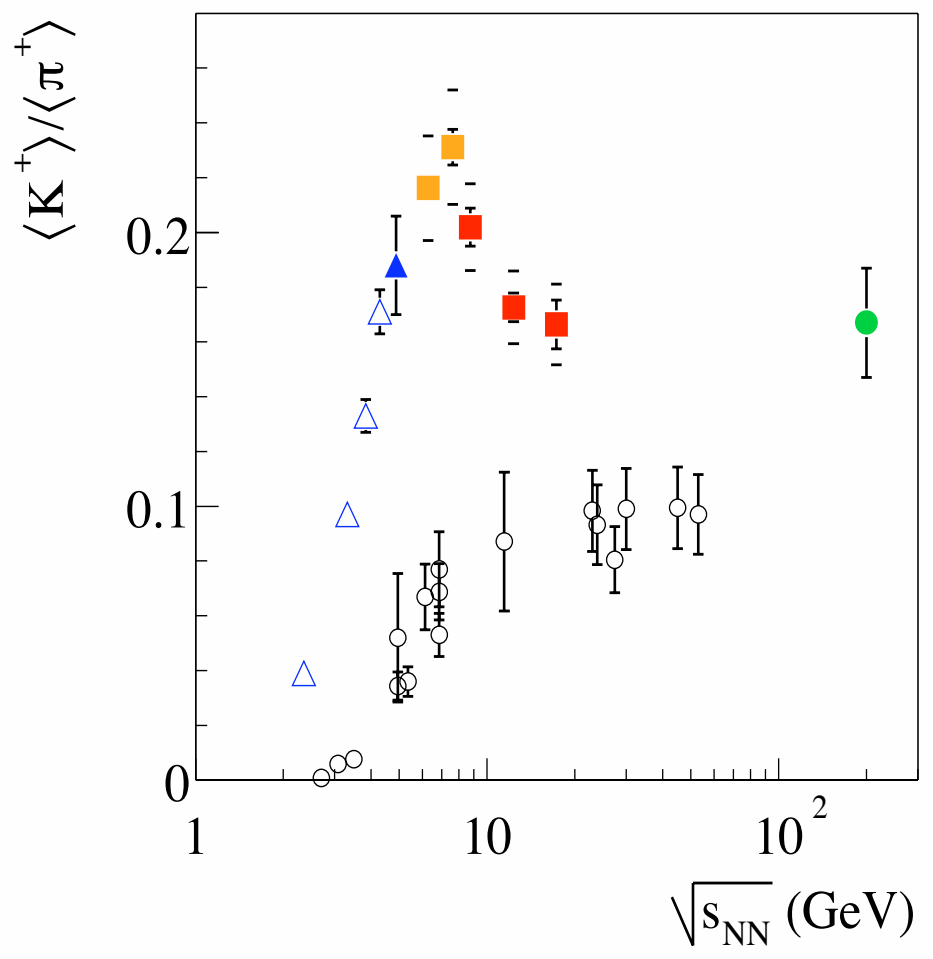

Figure 1: Energy dependence of full phase space $\left\langle K^{+}\right\rangle /\left\langle\pi^{+}\right\rangle$and $\left\langle K^{-}\right\rangle /\left\langle\pi^{-}\right\rangle$ratios in central $\mathrm{Pb}+\mathrm{Pb}$ $(\mathrm{Au}+\mathrm{Au})$ collisions. The results of NA49 are indicated by squares. The data for $p+p$ interactions are shown by open circles for comparison.

specific energy loss $(d E / d x)$ using a truncated mean algorithm. Details of the $d E / d x$ measurement can be found in [11] and in particular in [13]. As the event-by-event particle identification depends crucially on the stability of the $d E / d x$ measurement with respect to time, event multiplicity and possible backgrounds, only the energy loss of the track in the large volume Main-TPC's outside the magnetic field was used in this analysis. An average $d E / d x$ resolution $\sigma_{d E / d x}\langle d E / d x\rangle=3.9 \%$ is achieved for central $\mathrm{Pb}+\mathrm{Pb}$ collisions, independent of particle multiplicity. This resolution provides satisfactory separation between the different particle species above a minimum total momentum of $3 \mathrm{GeV} / c$. Figure 2 shows the phase space distribution of accepted pions, kaons and protons and illustrates a shifting cutoff for pions due to the particle identification requirements.

At all five available beam energies the $3.5 \%$ most central $\mathrm{Pb}+\mathrm{Pb}$ collisions were selected based on projectile spectator energy deposited in the NA49 forward calorimeter.

\section{Analysis Method}

Given the finite number of particles recorded for single $\mathrm{Pb}+\mathrm{Pb}$ collisions and the limited particle identification capabilities of a TPC in the relativistic rise region, it becomes crucial to make maximum use of the available information when constructing an estimator for the event-by-event 
particle ratios. In particular a simple counting of particles is not possible under these conditions. In this analysis a particle ratio estimation method closely related to that proposed in [14] is employed.

Combining the information from all events, the momentum distributions normalized to unity, $F_{m}(p)$, were determined for each particle species ( $m=$ kaons, pions, protons, electrons). We also evaluated the normalized probability density functions for the truncated mean energy loss, $f_{m}(\vec{p} ; d E / d x)$, as a function of particle momentum for each species. The relative yield of different particle species is characterized by parameters $\Theta_{m}$, such that $\sum_{m} \Theta_{m}=1$. These parameters were determined for every single event by maximizing the following likelihood function:

$$
L=\prod_{i=1}^{n}\left[\sum_{m} \Theta_{m} F_{m}\left(p_{i}\right) f_{m}\left(p_{i} ;(d E / d x)_{i}\right)\right] .
$$

Where $p_{i}$ denotes the observed momentum and $(d E / d x)_{i}$ the specific energy loss for each particle $i$ in the event.

For illustration, the distributions of the two studied particle ratios at 20,40 and $158 \mathrm{AGeV}$ are shown in figures 3 and 4. The relative width $\sigma$, defined as $\sigma=R M S / M e a n * 100$ [\%], of the measured event-by-event particle ratio distributions can be decomposed into three contributions:

1. Due to the finite number of particles produced and observed per event, the ratio of particle multiplicities measured event-by-event will exhibit statistical fluctuations with a width dictated by the individual particle multiplicities.

2. Due to non-ideal particle identification, these statistical fluctuations will be smeared by the experimental $d E / d x$ resolution and the event-by-event fitting procedure.

3. Superimposed on the background of statistical and experimental fluctuations we may observe genuine non-statistical fluctuations.

The contributions due to finite number fluctuations in the particle multiplicities and effects of detector resolution are estimated using a mixed event technique. Mixed events are constructed by randomly selecting measured particles from different events and combining them to artificial events, while reproducing the multiplicity distribution of the real events. By construction, mixed events have on average the same particle ratios as the real events, but no internal correlations. These mixed events are then subjected to the same fit procedure as the real events and the r.m.s width $q_{\text {nix }}$ of the mixed event particle ratio distribution is obtained. The contribution of dynamical fluctuations to the width of the distribution of particle ratios in data events can be estimated by subtracting the width of the mixed event distribution of particle ratios:

$$
\sigma_{\text {dyn }}=\operatorname{sign}\left(\sigma_{\text {data }}^{2}-\sigma_{\text {mixed }}^{2}\right) \sqrt{\left|\sigma_{\text {data }}^{2}-\sigma_{\text {mixed }}^{2}\right|}
$$

The systematic error of the fluctuation measurement is estimated by comparing the fluctuation signals obtained in two different analysis using varying sets of track quality cuts. Track samples selected with a stringent set of track quality cuts are compared to samples obtained with lose track cuts. The track selection using lose track quality cuts yields $15-20 \%$ more tracks per event to drive the event-by-event particle ratio estimation, but also increases the contamination of the track 
sample with non primary and background particles. The values of the fluctuation signal presented here are calculated as the arithmetic mean of both results.

Figure 3 shows the distribution of the event by event $K / \pi$ ratio ratio at 20,40 and $158 \mathrm{AGeV}$, the tight track selection on the left hand side of the panel and the loose selection on the right. Figure 4 shows the corresponding distributions for the $p / \pi$ ratio. Note that even though the absolute difference in width of data and mixed events is small, it can be measured with high precision given the large number of analyzed events. The significance of the measurement is reflected in the systematic error.

\section{Results}

The contribution from dynamical fluctuations obtained from equation 3.2 is plotted in figure 5 . The observed fluctuations of the $K / \pi$ ratio are positive and decrease with beam energy. In case of the $[p+\bar{p}] / \pi$ ratio, the width of the data distribution is smaller than the width of the distribution of mixed events. The dynamical fluctuations are negative. A negative fluctuation signal of the eventwise $[p+\bar{p}] / \pi$ ratio can be understood, if resonance decays into pions and protons are considered. The magnitude of the negative fluctuation signal in the $[p+\bar{p}] / \pi$ channel may be related to the relative contribution of resonance decay products in the final state of the collision.

In order to estimate the influence of trivial correlations in the event, like conservation laws and resonance decays, we analyze fluctuation signals of the two ratios considered in a string-hadronic cascade model UrQMD [15]. In this model, by construction, no fluctuations due to a potential phase transition are present. For this study large samples UrQMD events are generated at all five beam energies and then subjected to an acceptance filter modeling the NA49 detector system. The accepted final state particles were counted and the corresponding ratios were formed. The energy dependence of the event-by-event $[p+\bar{p}] / \pi$ ratio in UrQMD closely matches the energy dependence observed in the data, as shown in figure 5. This lends further support to interpreting the negative fluctuation signal as resulting from resonance decays.

In case of the fluctuations of the event wise $K / \pi$ ratio, the energy dependence of the signal cannot be reproduced in the cascade model. UrQMD gives an energy independent fluctuation signal. Since the relative contribution of resonances changes dramatically with incident beam energy [4], we conclude that in case of the $K / \pi$ ratio resonances do not give a significant contribution to the fluctuation signal. The finite fluctuation signal in the UrQMD model can be attributed to correlated particle production due to conservation laws.

Recently the STAR collaboration showed preliminary results on the event-by-event fluctuations of the $K / \pi$ ratio in Au+Au collisions at the Relativistic Heavy Ion Collider (RHIC) [16]. Using a similar experimental method they obtain dynamical fluctuations in the event wise $K / \pi$ ratio of $4.6 \%$ for the $5 \%$ most central Au+Au collisions at center of mass energies of $\sqrt{s_{N N}}=200$ $\mathrm{GeV}$ as $4.6 \%$ and $4.44 \%$ at $62.4 \mathrm{GeV}$. The distribution of the event-by-event $K / \pi$ ratio superimposed with the corresponding distribution for mixed events is shown in figure 6 . Given the collider setup and the geometrical acceptance of the STAR TPC, this measurement is limited to a region around midrapidity of two units of pseudorapidity $(|\eta|<1)$ with full coverage of the azimuthal angle $(-\pi<\phi<\pi)$. The particle identification is also based on the measured specific ionization 
in the TPC, but in this case in the $1 / \beta^{2}$ range of the Bethe Bloch curve allowing to separate pions and kaons in a momentum range from 100 to $600 \mathrm{MeV} / \mathrm{c}$.

The data in the RHIC energy range are within errors consistent with the two highest energy points obtained at the SPS as shown in figure 6. The fluctuation signal in the data taken at 158 $A \mathrm{GeV}$ incident energy was found to be consistent with calculations performed assuming a grand canonical ensemble without enforcing local conservation laws [17]. The fluctuation pattern is thus consistent with every event being a random sample from the identical thermal ensemble. The constant evolution towards higher beam energy indicates no significant deviation from this behavior.

Towards lower beam energies a steep increase of the fluctuation signal is observed as shown in the left panel of figure 5. This observation is consistent with many physics scenarios predicting the presence of a deconfining phase transition that could be observed at the lowest beam energies of the CERN SPS. The Statistical Model of the Early Stage that is able to describe the sharp nonmonotonic energy dependence of the $K^{+} / \pi^{+}$ratio would predict the presence of an extended mixed phase of confined and deconfined matter in this collision energy range, the would lead naturally to significant fluctuation of the event-by-event $K / \pi$ ratio. At higher incident energies the a sufficiently high energy density is achieved to produce a pure plasma phase, which leads to a constant, energy independent fluctuation pattern.

In order to interpret the energy dependence of the dynamical fluctuations in the event wise $K / \pi$ ratio we need to keep in mind the experimental fact of the changing acceptance of the measurements at different energies. The STAR measurements probe the midrapidity region, while the NA49 data is increasingly focused to forward rapidities. This change in kinematic coverage may lead to probing different physics processes driving the fluctuations event-by-event observables.

We would also like to add a conjecture from Stephanov [10] to this discussion, who pointed out that fluctuations of multiplicity ratios should be volume dependent and need to be scaled with $1 / N_{t}$ to obtain an intensive measure of matter properties. Applying this scaling to the NA49 data removes a significant fraction of the energy dependence. Resolving this issue will require further studies. On the experimental side the fluctuation signals should be studied within subsets of the given acceptance to test the scaling hypothesis. Also attempts will be made to extend that acceptance of the experimental setup by using more sophisticated analysis techniques. Theoretically more calculations are needed to study the effects of limited acceptance on potential sources of event-byevent fluctuations of particle ratios.

\section{Summary}

We have presented a study of the energy dependence of event-by-event fluctuations of the kaon to pion and proton to pion ratios ranging from the lowest CERN-SPS to the highest RHIC energies. A significant increase of the fluctuation signal of the kaon to pion ratio at 20 and $30 \mathrm{AGeV}$ is observed while it stays constant from the highest SPS energies out to the RHIC energy range. The increase of the signal goes significantly beyond the value seen in a hadronic cascade model, indicating the onset of a new source of fluctuations. The domain of increased coincides with the range where properties of the inclusive particle spectra also suggest the presence of phenomena possibly related to a deconfined phase in the early stage of the collision [4]. However further 
theoretical calculations will be needed to evaluate the relevance of this observation for a possible interpretation in the context of a deconfinement phase transition.

This work was supported by the US Department of Energy Grant DE-FG03-97ER41020/A000, the Bundesministerium fur Bildung und Forschung, Germany, the Polish State Committee for Scientific Research (2 P03B 130 23, SPB/CERN/P-03/Dz 446/2002-2004, 2 P03B 04123), the Hungarian Scientific Research Foundation (T032648, T032293, T043514), the Hungarian National Science Foundation, OTKA, (F034707), the Polish-German Foundation, and the Korea Research Foundation Grant (KRF-2003-070-C00015).

\section{References}

[1] M.Gaździcki and D.Röhrich,Z. Phys. C65 215 (1995)

M. Gaździcki and D. Röhrich, Z. Phys. C71 55 (1996) and references therein.

[2] M.Gaździcki, Z. Phys. C66 659 (1995).

[3] M.Gaździcki and M.I.Gorenstein Acta Phys. Polon. B30, 2705 (1999) and references therein.

[4] M.Gaździcki (for the NA49 collaboration) Preprint nucl-ex/0403023 (2004).

[5] S.V. Afanasiev et al. Phys. Rev. C66 157-166 (2002).

[6] R. Stock Nature 337 319-324 (1989).

[7] J.I. Kapusta and A. Mekjian Phys. Rev. D33 1304 (1986).

[8] M.Gazdzicki, M.I. Gorensteinand St.Mrowczynski Phys. Lett. B585 115 (2004).

[9] M.Stephanov Prog. Theor. Phys. Suppl. 153139 (2004).

[10] M.Stephanov, private communication.

[11] S.V.Afanasievet al. Nucl. Instr. and Meth. in Phys. Res. A430 210 (1999).

[12] S.V.Afanasiev et al. Phys. Rev. Lett. 861965 (2001).

[13] C.Roland, PhD Thesis University Frankfurt (1999).

[14] M.GazdzickiNucl. Instr. and Meth. in Phys. Res. $\mathbf{A 3 4 5} 148$ (1994).

[15] M.Bleicher et al. J. Phys. G25 1859 (1999).

[16] S.Das Preprint nucl-ex/0503023 and these proceedings.

[17] S.Jeon and V.Koch Phys. Rev. Lett. 835435 (1999). 

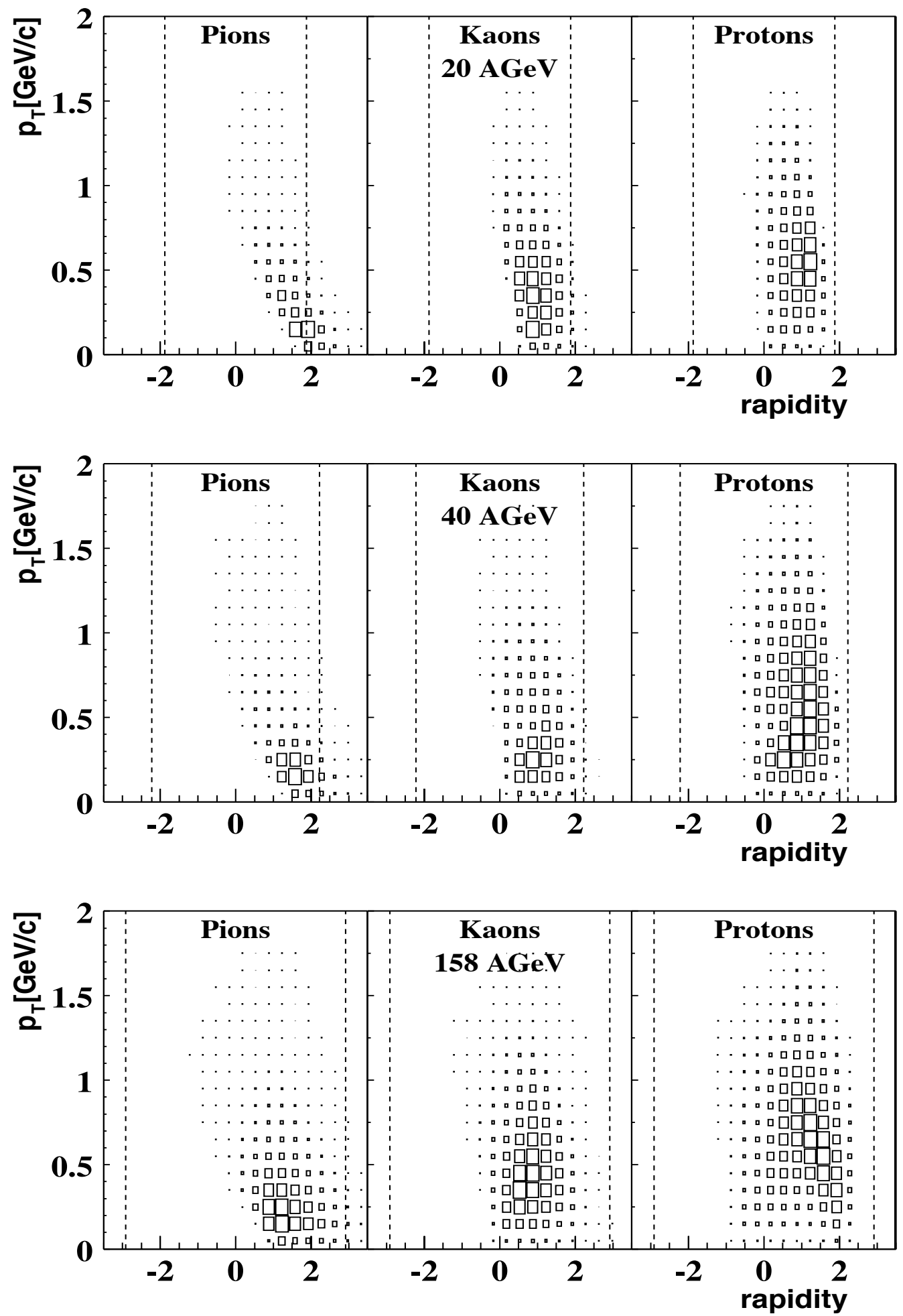

Figure 2: Phase-space distribution of accepted charged particles at $20 \mathrm{AGeV}$ (upper panel) and $158 \mathrm{AGeV}$ (lower panel). From left to right pions, kaons and protons are shown. 

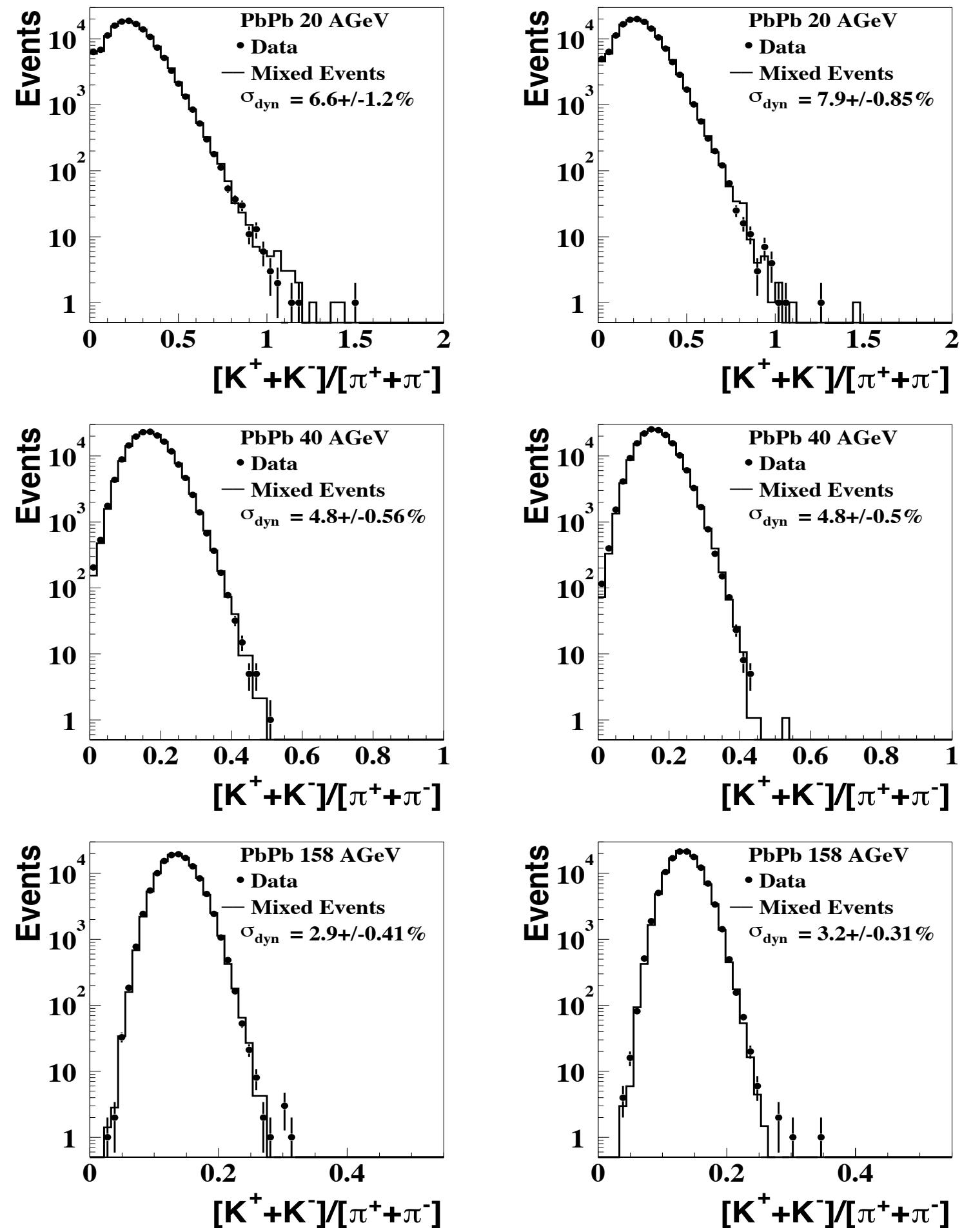

Figure 3: Distributions of the event-by-event $\left[K^{+}+K^{-}\right] /\left[\pi^{+}+\pi^{-}\right]$ratio for data (points) and mixed events (histogram). No acceptance correction was applied to the particle ratios shown. The left hand column of figures shows the distributions obtained with a tight track selection. The loose selection is shown on the right. 

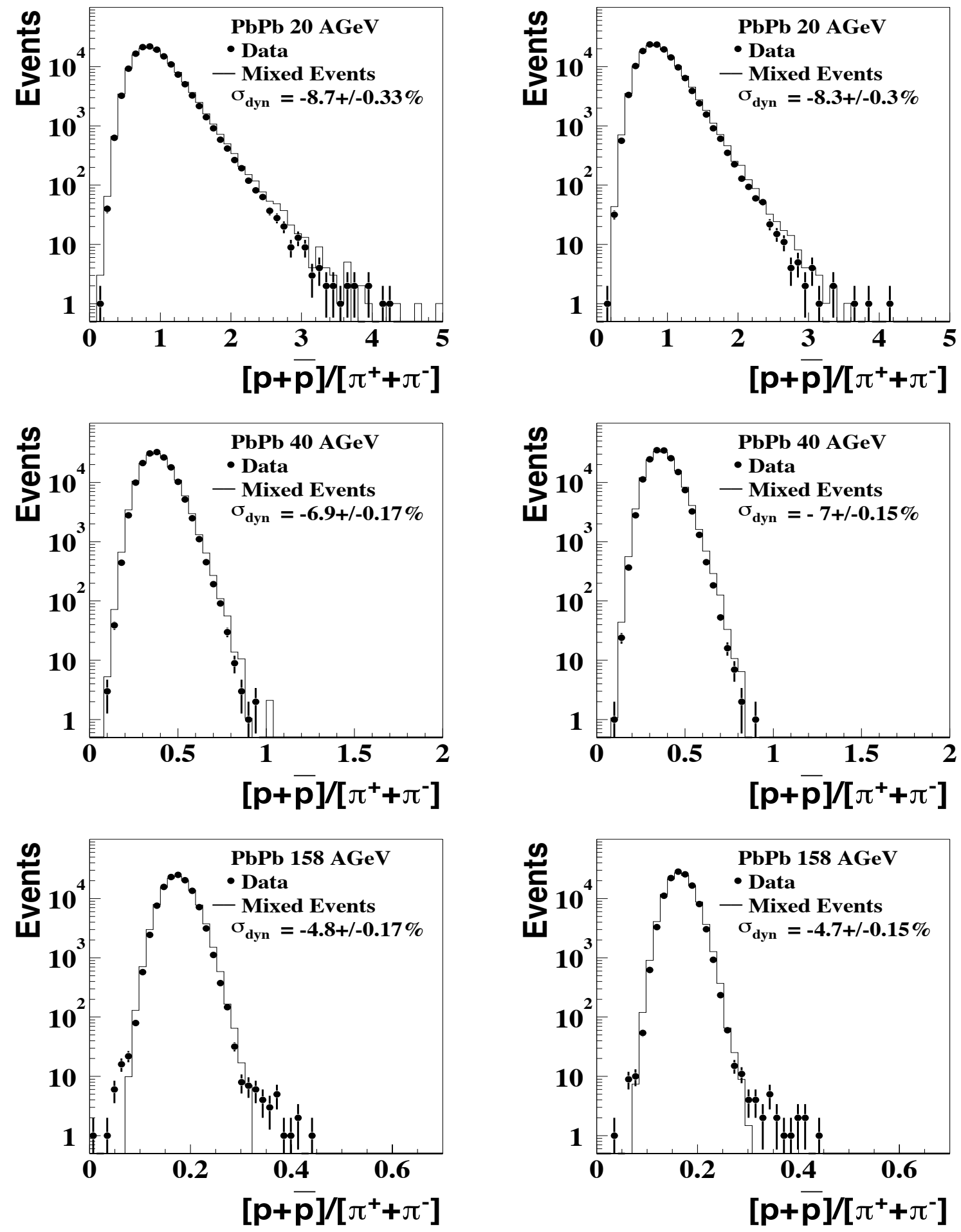

Figure 4: Distributions of the event-by-event $[p+\bar{p}] /\left[\pi^{+}+\pi^{-}\right]$ratio for data (points) and mixed events (histogram). No acceptance correction was applied to the particle ratios shown.The left hand column of figures shows the distributions obtained with a tight track selection. The loose selection is shown on the right. 

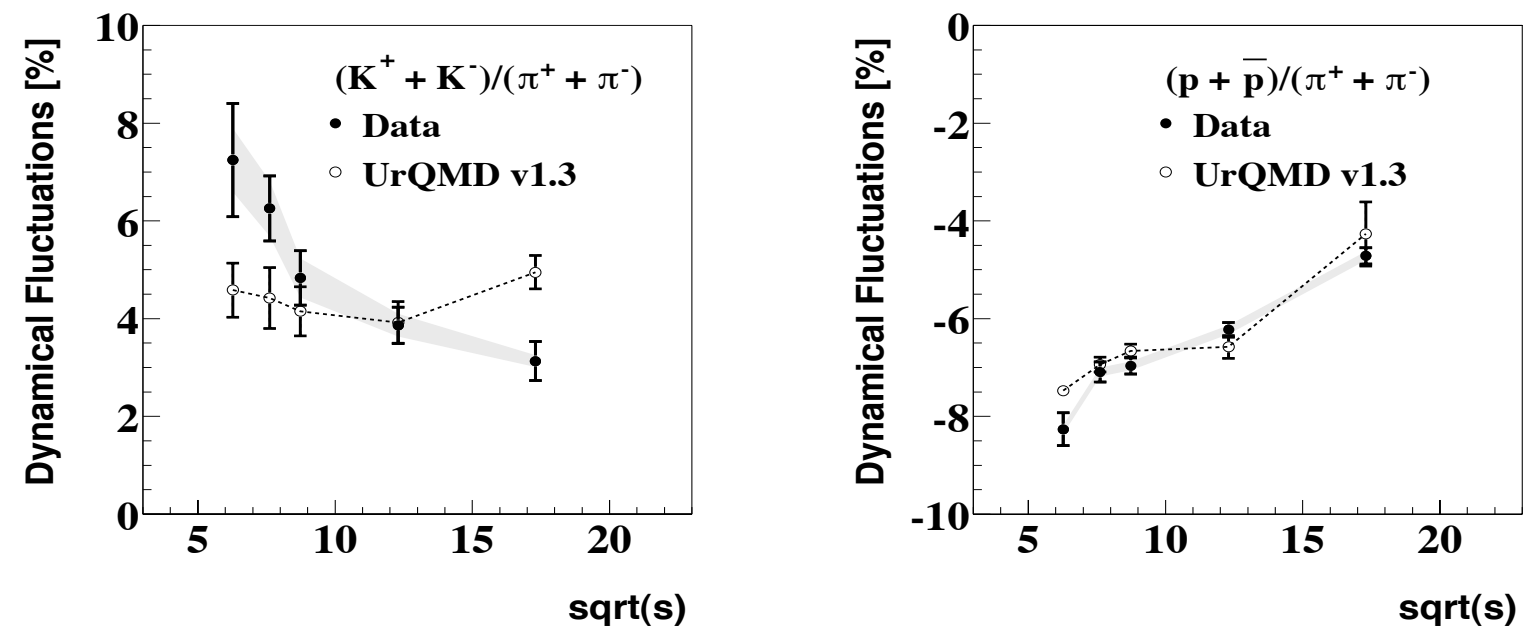

Figure 5: Energy dependence of the event-by-event fluctuation signal of the $\left[K^{+}+K^{-}\right] /\left[\pi^{+}+\pi^{-}\right]$ratio (left panel) and the $[p+\bar{p}] /\left[\pi^{+}+\pi^{-}\right]$ratio (right panel). The systematic errors of the measurements are shown as gray bands.
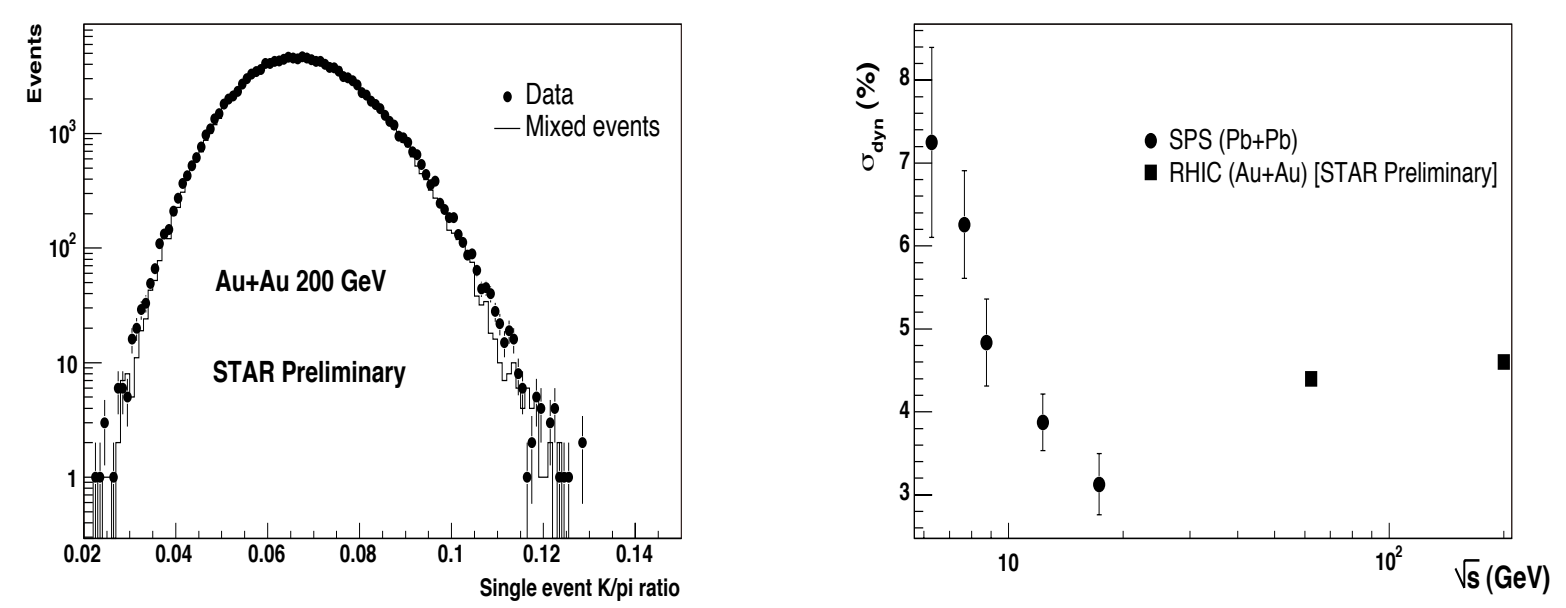

Figure 6: Preliminary data obtained by the STAR collaboration. Left panel: Distribution of $K / \pi$ ratio from data and mixed events for $\mathrm{Au}+\mathrm{Au}$ at $200 \mathrm{GeV}$. Right panel: Energy dependence of the event-by-event fluctuation signal of the $\left[K^{+}+K^{-}\right] /\left[\pi^{+}+\pi^{-}\right]$ratio. 